Canad. Math. Bull. Vol. 20 (1), 1977

\title{
REMARQUE SUR UN ARTICLE DE T. M. APOSTOL
}

\author{
PAR \\ JEAN-MARIE DE KONINCK ET ARMEL MERCIER
}

1. Introduction. Dans un récent article [1], T. M. Apostol obtient une identité liant $\sum_{n=1}^{\infty} f(n) \mu(n) n^{-s}$ et $\sum_{n=1}^{\infty} f(n) \mu(n) \mu(p, n) n^{-s}$, où $f$ est une fonction complètement multiplicative, $|f(n)| \leq 1$, et $p$ est un nombre premier. Il utilise cette identité pour prouver que

$$
\sum_{\substack{n=1 \\ n=0(p)}}^{\infty} \frac{\mu(n)}{n}=0 \quad \text { et } \quad \sum_{\substack{n=1 \\ n \neq 0(p)}}^{\infty} \frac{\mu(n)}{n}=0,
$$

pour tout nombre premier $p$. Par la suite, T. M. Apostol obtenait d'autres identités [2].

Nous établirons ici quelques identités pour les séries de Dirichlet et nous les utiliserons pour trouver l'ordre de grandeur de $\sum_{\substack{n \leq x \\ n=0\left(p_{0}\right)}} f(n)$ et $\sum_{\substack{n \leq x \\ n \equiv 0\left(p_{0}\right)}}^{\prime}[1 / f(n)]$ pour une certaine classe de fonctions additives, $\underset{\substack{n \leq x \\ n=0\left(p_{0}\right)}}{\left(\sum_{0}\right.}[1 / f(n)]$ signifie que la somme parcourt tous les $n \leq x, n \equiv 0\left(p_{0}\right)$, tels que $\left.f(n) \neq 0\right)$, ainsi que l'ordre de grandeur de $\sum_{\substack{n \leq x \\ n \equiv 0\left(p_{0}\right)}} \theta(n) f(n)$ où $\theta(n)$ et $f(n)$ appartiennent respectivement à une classe de fonctions multiplicatives et additives.

2. Notation. On dénote par $\mu(m, n)$ la fonction de Möbius évaluée au p.g.c.d. entre $m$ et $n, m, n \in \mathbb{N}$. Lorsqu'il existe, nous désignerons par $\sigma_{a}$ l'abscisse de convergence absolue des séries de Dirichlet.

\section{Enoncé des principaux résultats}

THÈORÈME 1. Soit $f$ une fonction multiplicative telle que $\sum_{n=1}^{\infty} f(n) n^{-s}$ converge absolument pour tout nombre complexe $s=\sigma+i t, \sigma>\sigma_{a}$. Alors pour tout entier $k \in \mathbb{N}$ et pour tout nombre complexe $s=\sigma+i t, \sigma>\sigma_{a}$, nous avons

$$
\sum_{n=1}^{\infty} \frac{f(n) \mu(n) \mu(k, n)}{n^{s}}=\beta(k, s ; f) \sum_{n=1}^{\infty} \frac{\mu(n) f(n)}{n^{s}},
$$

où

$$
\beta(k, s ; f)=\prod_{p \mid k}\left(\frac{1+f(p) p^{-s}}{1-f(p) p^{-s}}\right) .
$$

Received by the editors January 27,1977 in revised form. 
De plus, si $|f(p)|<p^{c}$, c étant un nombre réel plus petit ou égal à $\sigma_{a}$, et si $\sum_{n=1}^{\infty} f(n) \mu(n) n^{-s}$ converge pour $\sigma \geq c$, alors (1) est valide pour $\sigma \geq c$.

Preuve. La preuve se fait de la même façon que celle utilisée par T. M. Apostol dans [2].

THÉORÈME 2. Soit $f$ une fonction multiplicative telle que $\sum_{n=1}^{\infty} f(n) n^{-s}$ converge absolument pour tout nombre complexe $s=\sigma+i t, \sigma>\sigma_{a}$. Alors, pour tout nombre premier $p_{0}$, et pour $\sigma>\sigma_{a}$, on $a$

$$
\sum_{\substack{n=1 \\ n \equiv 0\left(p_{0}\right)}}^{\infty} \frac{f(n)}{n^{s}}=\left(\frac{f\left(p_{0}\right)}{p_{0}^{s}}+\frac{f\left(p_{0}^{2}\right)}{p_{0}^{2 s}}+\cdots\right)\left(1+\frac{f\left(p_{0}\right)}{p_{0}^{s}}+\frac{f\left(p_{0}^{2}\right)}{p_{0}^{2 s}}+\cdots\right)^{-1} \sum_{n=1}^{\infty} \frac{f(n)}{n^{s}}
$$

De plus, si $|f(n)|<n^{c}$, c étant un nombre réel plus petit ou égal à $\sigma_{a}$, et si $\sum_{n=1}^{\infty} f(n) n^{-s}$ converge pour $\sigma \geq c$, alors (2) est valide pour $\sigma \geq c$.

Remarque. (i) Si $f$ est une fonction complètement multiplicative, alors pour tout entier $k \in \mathbb{N}$, on a

$$
\sum_{\substack{n=1 \\ n=0(k)}}^{\infty} \frac{f(n)}{n^{s}}=\sum_{n=1}^{\infty} \frac{f(n k)}{(n k)^{s}}=\frac{f(k)}{k^{s}} \sum_{n=1}^{\infty} \frac{f(n)}{n^{s}}
$$

(ii) Si $f$ est une fonction multiplicative, alors pour tout entier $\mathrm{k} \in \mathbb{N}$, on a pour $\sigma>\sigma_{a}$

$$
\begin{aligned}
\sum_{\substack{n=1 \\
n=0(k)}}^{\infty} \frac{f(n)}{n^{s}}= & \sum_{n=1}^{\infty} \frac{f(n k)}{(n k)^{s}}=\frac{1}{k^{s}} \sum_{d \mid k} \sum_{\substack{n=1 \\
(k, n)=d}}^{\infty} \frac{f(n k)}{n^{s}} \\
= & \frac{f(k)}{k^{s}} \prod_{p \mid k}\left(1+\frac{f(p)}{p^{s}}+\frac{f\left(p^{2}\right)}{p^{2 s}}+\cdots\right)^{-1} \\
& \times \sum_{n=1}^{\infty} \frac{f(n)}{n^{s}}+\frac{1}{k^{s}} \sum_{\substack{d \mid k \\
d>1}} \sum_{\substack{n=1 \\
(k, n)=d}}^{\infty} \frac{f(n k)}{n^{s}}
\end{aligned}
$$

(iii) Si l'on connaît $\sum_{\substack{n=1 \\ n \equiv 0(k)}}^{\infty}\left[f(n) / n^{s}\right]$, il est facile d'établir une identité $\operatorname{pour} \sum_{\substack{n=1 \\ n \neq 0(k)}}^{\infty}\left[f(n) / n^{s}\right]$ puisque

$$
\sum_{\substack{n=1 \\ n \equiv 0(k)}}^{\infty} \frac{f(n)}{n^{s}}+\sum_{\substack{n=1 \\ n \neq 0(k)}}^{\infty} \frac{f(n)}{n^{s}}=\sum_{n=1}^{\infty} \frac{f(n)}{n^{s}}
$$


4. Preuve du Théorème 2. Pour $\sigma>\sigma_{a}$, on a

$$
\begin{aligned}
\sum_{\substack{n=1 \\
n=0\left(p_{0}\right)}}^{\infty} \frac{f(n)}{n^{s}} & =\sum_{\substack{n=1 \\
\left(p_{0}, n\right)=1}}^{\infty} \frac{f\left(p_{0} n\right)}{\left(p_{0} n\right)^{s}}+\sum_{\substack{n=1 \\
\left(p_{0}, n\right)=1}}^{\infty} \frac{f\left(p_{0}^{2} n\right)}{\left(p_{0}^{2} n\right)^{s}}+\cdots \\
& =\left(\frac{f\left(p_{0}\right)}{p_{0}^{s}}+\frac{f\left(p_{0}^{2}\right)}{p_{0}^{2 s}}+\cdots\right) \sum_{\substack{n=1 \\
\left(p_{0}, n\right)=1}}^{\infty} \frac{f(n)}{n^{s}} \\
& =\left(\frac{f\left(p_{0}\right)}{p_{0}^{s}}+\frac{f\left(p_{0}^{2}\right)}{p_{0}^{2 s}}+\cdots\right)\left(1+\frac{f\left(p_{0}\right)}{p_{0}^{s}}+\frac{f\left(p_{0}^{2}\right)}{p_{0}^{2 s}}+\cdots\right)^{-1} \sum_{n=1}^{\infty} \frac{f(n)}{n^{s}}
\end{aligned}
$$

Si $|f(n)|<n^{c}, c \leq \sigma_{a}$, alors pour $\sigma \geq c$,

$$
\sum_{\alpha=1}^{\infty} \frac{f\left(p_{0}^{\alpha}\right)}{\left(p_{0}^{\alpha}\right)^{s}}
$$

est une série de Dirichlet absolument convergente. Donc

$$
\left(\sum_{\alpha=0}^{\infty} \frac{f\left(p_{0}^{\alpha}\right)}{p_{0}^{\alpha s}}\right)\left(\sum_{\alpha=1}^{\infty} \frac{f\left(p_{0}^{\alpha}\right)}{p_{0}^{\alpha s}}\right)
$$

est une série de Dirichlet qui converge absolument. Or, si $\sum_{n=1}^{\infty} f(n) n^{-s}$ converge pour $\sigma \geq c$, alors (2) est aussi valide pour $\sigma \geq c$ puisque $\sum_{\substack{n=1 \\ n=0\left(p_{0}\right)}}^{\infty}\left[f(n) / n^{s}\right]$ est le produit d'une série de Dirichlet absolument convergente et d'une série de Dirichlet convergente.

Remarque. Dorénavant, pour toutes fonctions multiplicatives, nous supposons que leurs séries de Dirichlet correspondantes convergent absolument pour $\sigma>\sigma_{a}$.

\section{Applications}

THÉORÈME 3. Soit $f$ une fonction additive telle que $f(p)$ est indépendante de $p$ et telle

$$
\sum_{p} \frac{1}{f(p)}\left(\frac{f\left(p^{2}\right)}{p^{2}}+\frac{f\left(p^{3}\right)}{p^{3}}+\cdots\right)\left(1-\frac{1}{p}\right) \quad \text { et } \sum_{\alpha=1}^{\infty} \frac{f\left(p_{0}^{\alpha}\right)}{p_{0}^{\alpha}}
$$

convergent, alors

$$
\sum_{\substack{n \leq x \\ n=0\left(p_{0}\right)}} f(n)=\frac{f(p)}{p_{0}} x \log \log x+C x+O\left(\frac{x}{\log x}\right),
$$


où $p_{0}$ est un nombre premier fixe et

$$
\begin{aligned}
C= & \left(1-\frac{1}{p_{0}}\right)^{2}\left(\frac{f\left(p_{0}\right)}{p_{0}}+\frac{f\left(p_{0}^{2}\right)}{p_{0}^{2}}+\cdots\right)+\frac{f(p)}{p_{0}} \\
& \times \sum_{p}\left\{\log \left(1-\frac{1}{p}\right)+\left(\frac{1}{p}+\frac{f\left(p^{2}\right) / f(p)}{p^{2}}+\frac{f\left(p^{3}\right) / f(p)}{p^{3}}+\cdots\right)\left(1-\frac{1}{p}\right)\right\}+\frac{\gamma f(p)}{p_{0}}
\end{aligned}
$$

( $\gamma$ étant la constante d'Euler).

Preuve. Par le Théorème 2,

$$
\begin{aligned}
& \sum_{\substack{n=1 \\
n \equiv\left(p_{0}\right)}}^{\infty} \frac{t^{f(n)}}{n^{s}}=\left(\frac{t^{f\left(p_{0}\right)}}{p_{0}^{s}}+\frac{t^{f\left(p_{0}{ }^{2}\right)}}{p_{0}^{2 s}}+\cdots\right)\left(1+\frac{t^{f\left(p_{0}\right)}}{p_{0}^{s}}+\cdots\right)^{-1} \\
& \times \prod_{p}\left(1+\frac{t^{f(p)}}{p^{s}}+\frac{t^{f\left(p^{2}\right)}}{p^{2 s}}+\cdots\right)
\end{aligned}
$$

pour $t \in \mathbb{C},|t| \leq 1$, et $\operatorname{Re} s>\sigma_{a}$.

Soient $u=t^{f(p)}$ et

alors

$$
w=\frac{u^{f\left(p_{0}\right) / f(p)}}{p_{0}^{s}}+\frac{u^{f\left(p_{0}^{2}\right) / f(p)}}{p_{0}^{2 s}}+\cdots
$$

$$
\sum_{\substack{n=1 \\ n=0\left(p_{0}\right)}}^{\infty} \frac{u^{f(n) / f(p)}}{n^{s}}=g(s, u) \zeta^{u}(s)
$$

( $\zeta$ étant la fonction Zeta de Riemann) où

$$
g(s, u)=w(1+w)^{-1} \prod_{p}\left(1+\frac{u}{p^{s}}+\frac{u^{f\left(p^{2}\right) / f(p)}}{p^{2 s}}+\cdots\right) \prod_{p}\left(1-\frac{1}{p^{s}}\right)^{u} .
$$

D'où d'après un théorème de Selberg [6], nous trouvons

$$
\sum_{\substack{n \leq x \\ n=0\left(p_{0}\right)}} u^{f(n) / f(p)}=\frac{g(1, u)}{\Gamma(u)} x \log ^{u-1} x+R(x, u),
$$

où $R(x, u)$ est $O\left(x \log ^{u-2} x\right)$ uniformément pour $|u| \leq 1$. Derivant par rapport à $u$ tout en utilisant l'inégalité de Cauchy pour estımer $R^{\prime}(x, u)$, et en posant $u=1$, on trouve le résultat demandé.

THÉORÈME 4. Soit $p_{0}$ un nombre premier fixe, alors

où

$$
\sum_{\substack{n \leq x \\ n \equiv 0\left(p_{0}\right)}} \Omega(n)=\frac{1}{p_{0}} x \log \log x+C_{1} x+O\left(\frac{x}{\log x}\right),
$$

$$
C_{1}=\frac{1}{p_{0}}\left[\sum_{p}\left(\log \left(1-\frac{1}{p}\right)+\frac{1}{p-1}\right)+\gamma+1\right] .
$$


Preuve. Immédiat d'après le Théorème 3.

THÉORÈME 5. Soit $p_{0}$ un nombre premier fixe, alors

où

$$
\sum_{\substack{n \leq x \\ n=0\left(p_{0}\right)}} \omega(n)=\frac{1}{p_{0}} x \log \log x+C_{2} x+O\left(\frac{x}{\log x}\right),
$$

$$
C_{2}=\frac{1}{p_{0}}\left[\sum_{p}\left(\log \left(1-\frac{1}{p}\right)+\frac{1}{p}\right)+\left(1-\frac{1}{p_{0}}\right)+\gamma\right] .
$$

Preuve. Immédiat d'après le Théorème 3.

Avant d'énoncer le Théorème 6, nous écrirons les définitions suivantes qui furent données pour la première fois par le premier auteur (voir [5]).

DÉfINITION 1. Soit $S$ l'ensemble de toutes les fonctions arithmétiques $f$ à valeurs réelles qui satisfont

(1) $f(n) \neq 0 \Rightarrow f(n) \geq 1$ pour tout entier $n \geq 1$.

(2) $\sum_{\substack{n \leq x \\ f(n)=0}} 1=O\left(\frac{x}{\log x}\right)$.

Définition 2. Etant donné $\alpha$ (entier positif arbitraire), soit $S_{\alpha}$ l'ensemble de toutes les fonctions $f \in S$ pour lesquelles $t^{f(n)}=a_{t}(n)$ satisfait les conditions du théorème de Selberg (voir [6]) avec $B=1$ et

$$
D(t)=\frac{g(1, t)}{\Gamma(t)} \in C^{\alpha+1}[0,1] .
$$

Définttion 3. Etant donné $f \in S_{\alpha}$, soit $D(t)$ la fonction correspondante de la Définition 2 , alors pour $t \in(0,1]$, on pose

$$
B_{i}(t)=\left[\left(t^{f\left(p_{0}\right)}+\frac{t^{f\left(p_{0}{ }^{2}\right)}}{p_{0}}+\cdots\right)\left(1+\frac{t^{f\left(p_{0}\right)}}{p_{0}}+\frac{t^{f\left(p_{0}{ }^{2}\right)}}{p_{0}^{2}}+\cdots\right)^{-1} \frac{D(t)}{t}\right]^{(i-1)}
$$

(dérivée $(i-1)$ ième, et $A_{i}(t)=(-1)^{i-1} B_{i}(t), i=1,2, \ldots \alpha+2$. Nous noterons $A_{i}(1)=A_{i}$.

THÉORÈME 6. Soit $f \in S_{\alpha}$, alors

$$
\sum_{\substack{n \leq x \\ n=0\left(p_{0}\right)}} \frac{1}{f(n)}=\frac{x}{p_{0}} \sum_{i=1}^{\alpha} \frac{A_{i}}{(\log \log x)} i+O\left(\frac{x}{(\log \log x)^{\alpha+1}}\right),
$$

où $p_{0}$ est un nombre premier fixe. 
Preuve. En utilisant le Théorème 2 pour $\sigma>\sigma_{a}$, on a

$$
\begin{aligned}
\sum_{\substack{n=1 \\
n=0\left(p_{0}\right)}}^{\infty} \frac{t^{f(n)}}{n^{s}}= & \left(\frac{t^{f\left(p_{0}\right)}}{p_{0}^{s}}+\frac{t^{f\left(p_{0}{ }^{2}\right)}}{p_{0}^{2 s}}+\cdots\right)\left(1+\frac{t^{f\left(p_{0}\right)}}{p_{0}^{s}}+\frac{t^{f\left(p_{0}{ }^{2}\right)}}{p_{0}^{2 s}}+\cdots\right)^{-1} \\
& \times \sum_{n=1}^{\infty} \frac{t^{f(n)}}{n^{s}} .
\end{aligned}
$$

Or $f \in S_{\alpha}$, d'où

$$
\sum_{\substack{n \leq x \\ n=0\left(p_{0}\right)}} t^{f(n)}=\frac{1}{p_{0}}\left(t^{f\left(p_{0}\right)}+\frac{t^{f\left(p_{0}\right)}}{p_{0}}+\cdots\right)\left(1+\frac{t^{f\left(p_{0}\right)}}{p_{0}^{2}}+\cdots\right)^{-1} D(t) x \log ^{t-1} x+R(x, t),
$$

où

$$
R(x, t)=O\left(x \log ^{t-2} x\right) .
$$

Le reste de la preuve se fait de la même façon què celle utilisée dans [5].

THÉORÈME 7

où

$$
\sum_{\substack{n \leq x \\ n=0\left(p_{0}\right)}}^{\prime} \frac{1}{\omega(n)}=\frac{x}{p_{0}} \sum_{i=1}^{\alpha} \frac{a_{i}}{(\log \log x)^{i}}+O\left(\frac{x}{(\log \log x)^{\alpha+1}}\right)
$$

$$
\begin{aligned}
& a_{1}=1 \\
& a_{2}=\frac{1}{p_{0}}-\sum_{p}\left(\log \left(1-\frac{1}{p}\right)+\frac{1}{p}\right)-\gamma,
\end{aligned}
$$

et tous les autres $a_{i}$ sont calculables.

Preuve. Immédiat d'après le Théorème 6.

THÉORÈME 8. Soit $\theta$ une fonction multiplicative telle que $\theta(p)$ est indépendante de $p$ et $\theta(p) \neq 0,-1,-2, \ldots$, et soit $f$ une fonction additive telle que $f(p)$ est indépendante de p. Si

$$
\sum_{p}\left\{\log \left(1-\frac{1}{p}\right)+\frac{\frac{1}{p}+\frac{1}{\theta(p) f(p)}\left(\frac{\theta\left(p^{2}\right) f\left(p^{2}\right)}{p^{2}}+\frac{\theta\left(p^{3}\right) f\left(p^{3}\right)}{p^{3}}+\cdots\right)}{1+\frac{\theta(p)}{p}+\frac{\theta\left(p^{2}\right)}{p^{2}}+\cdots}\right\}
$$

converge, alors

$$
\sum_{n \leq x} \theta(n) f(n)=C x \log ^{\theta(p)-1} x \log \log x+C_{1} x \log ^{\theta(p)-1} x+O\left(x \log ^{\theta(p)-2} x\right),
$$


où

$$
\begin{aligned}
C= & \theta(p) f(p) \prod_{p}\left(1-\frac{1}{p}\right)^{\theta(p)} \prod_{p}\left(1+\frac{\theta(p)}{p}+\frac{\theta\left(p^{2}\right)}{p^{2}}+\cdots\right) / \Gamma(\theta(p)), \\
C_{1}= & \frac{C}{\Gamma(\theta(p))}\left[\sum_{p}\left\{\begin{array}{c}
\left.\log \left(1-\frac{1}{p}\right)+\frac{\frac{1}{p}+\frac{1}{\theta(p) f(p)}\left(\frac{\theta\left(p^{2}\right) f\left(p^{2}\right)}{p^{2}}+\frac{\theta\left(p^{3}\right) f\left(p^{3}\right)}{p^{3}}+\cdots\right)}{1+\frac{\theta(p)}{p}+\frac{\theta\left(p^{2}\right)}{p^{2}}+\cdots}\right\} \\
\\
\end{array}\right] \Gamma(\theta(p))-\Gamma^{\prime}(\theta(p))\right] .
\end{aligned}
$$

Preuve. Pour $\sigma>\sigma_{a}$,

$$
\sum_{n=1}^{\infty} \frac{\theta(n) t^{f(n)}}{n^{s}}=\prod_{p}\left(1+\frac{\theta(p) t^{f(p)}}{p^{s}}+\frac{\theta\left(p^{2}\right) t^{f\left(p^{2}\right)}}{p^{2 s}}+\cdots\right)
$$

pour $|t| \leq 1$. Soit $u=\theta(p) t^{f(p)}$, alors

$$
\begin{aligned}
\sum_{n=1}^{\infty} \frac{\theta(n)\left(\frac{u}{\theta(p)}\right)^{f(n) / f(p)}}{n^{s}} & =\prod_{p}\left(1+\frac{u}{p^{s}}+\frac{\theta\left(p^{2}\right)\left(\frac{u}{\theta(p)}\right)^{f\left(p^{2}\right) / f(p)}}{p^{2 s}}+\cdots\right) \\
& =\zeta^{u}(s) \prod_{p}\left(1-\frac{1}{p^{s}}\right)^{u} \prod_{p}\left(1+\frac{u}{p^{s}}+\cdots\right) \\
& =\zeta^{u}(s) g(s, u)
\end{aligned}
$$

D'où d'après un théorème de Selberg [6], on a

$$
\sum_{n \leq x} \theta(n)\left(\frac{u}{\theta(p)}\right)^{f(n) / f(p)}=\frac{g(1, u)}{\Gamma(u)} x \log ^{u-1} x+R(x, u),
$$

où $R(x, u)$ est $O\left(x \log ^{u-2} x\right)$ uniformément pour $|t| \leq 1$. Dérivant par rapport à $u$ tout en utilisant l'inégalité de Cauchy pour estimer $R^{\prime}(x, u)$, et en posant $t=1$, on trouve le résultant demandé.

THÉORÈME 9. Soit $d(n)$ le nombre de diviseurs de $n$, alors

$$
\sum_{n \leq x} d(n) \omega(n)=2 x \log x \log \log x+C_{1} x \log x+O(x),
$$

où

$$
C_{1}=2\left(\sum_{p}\left\{\log \left(1-\frac{1}{p}\right)+\left(\frac{1}{p}+\frac{3}{2 p^{2}}+\frac{4}{2 p^{3}}+\cdots\right)\left(1-\frac{1}{p}\right)^{2}\right\}-\Gamma^{\prime}(2)\right) .
$$


Preuve. Immédiat d'après le Théorème 8.

THÉORÈme 10. Avec les mêmes hypothèses du Théorème 8 sur les fonctions $\theta$ et $f$, et supposons de plus que $\sum_{\alpha=1}^{\infty}\left[\theta\left(p_{0}^{\alpha}\right) / p_{0}^{\alpha}\right]$ converge vers au nombre $\neq-1$, alors

$$
\sum_{\substack{n \leq x \\ n=0\left(p_{0}\right)}} \theta(n) f(n)=C^{*} x \log ^{\theta(p)} x^{-1} \log \log x+C_{1}^{*} x \log ^{\theta(p)} x^{-1}+O\left(x \log ^{\theta(p)-2} x\right)
$$

où

$$
C^{*}=C\left(\frac{\theta\left(p_{0}\right)}{p_{0}}+\frac{\theta\left(p_{0}^{2}\right)}{p_{0}^{2}}+\cdots\right)\left(1+\frac{\theta\left(p_{0}\right)}{p_{0}}+\frac{\theta\left(p_{0}^{2}\right)}{p_{0}^{2}}+\cdots\right)^{-1}
$$

(C étant la constante définie dans l'énoncé du Théorème 8) et $C_{1}^{*}$ étant une constante qu'on peut déterminer ( $p_{0}$ est un nombre premier arbitraire).

Preuve. Puisque $\theta(n) t^{f(n)}$ est une fonction multiplicative, alors par le Théorème 2, pour $\sigma>\sigma_{a}$,

$$
\begin{aligned}
& \sum_{\substack{n=1 \\
n=0\left(p_{0}\right)}}^{\infty} \frac{\theta(n) t^{f(n)}}{n^{s}}=\left(\frac{\theta\left(p_{0}\right) t^{f\left(p_{0}\right)}}{p_{0}^{s}}+\frac{\theta\left(p_{0}^{2}\right) t^{f\left(p_{0}{ }^{2}\right)}}{p_{0}^{2 s}}+\cdots\right) \\
& \times\left(1+\frac{\theta\left(p_{0}\right) t^{f\left(p_{0}\right)}}{p_{0}^{s}}+\cdots\right)^{-1} \sum_{n=1}^{\infty} \frac{\theta(n) t^{f(n)}}{n^{s}} .
\end{aligned}
$$

En utilisant le même procédé que celui du Théorème 8 on obtient le résultat.

THÉORÈME 11

$$
\sum_{\substack{n \leq x \\ n=0\left(p_{0}\right)}} d(n) \omega(n)=\left(\frac{2 p_{0}-1}{p_{0}^{2}}\right) 2 x \log x \log \log x+C x \log x+O(x),
$$

où

$$
C=\frac{\left(2 p_{0}-1\right)\left(p_{0}-1\right)^{2}}{p_{0}^{4}}+\frac{2 p_{0}-1}{p_{0}^{2}} C_{1},
$$

$C_{1}$ étant la constante définie dans le Théorème 9.

Preuve. Immédiat d'après le Théorème 10.

THÉORÈME 12. Soit $\theta$ une fonction multiplicative telle que $\theta(p)$ est indépendante de $p$ et $\theta(p) \neq 0,-1,-2, \ldots$ Si $\sum_{\alpha=1}^{\infty}\left[\theta\left(p_{0}^{\alpha}\right) / p_{0}^{\alpha}\right]$ converge vers un nombre $\neq-1$, alors

$$
\sum_{\substack{n \leq x \\ n=0\left(p_{0}\right)}} \theta(n) \sim\left(\frac{\theta\left(p_{0}\right)}{p_{0}}+\frac{\theta\left(p_{0}^{2}\right)}{p_{0}^{2}}+\cdots\right)\left(1+\frac{\theta\left(p_{0}\right)}{p_{0}}+\frac{\theta\left(p_{0}^{2}\right)}{p_{0}^{2}}+\cdots\right)^{-1} \sum_{n \leq x} \theta(n)
$$

où $p_{0}$ est un nombre premier fixe. 
Preuve. Posant $u=\theta(p)$ dans l'équation (3) du Théorème 8, on obtient

$$
\sum_{n \leq x} \theta(n)=\frac{g(1, \theta(p))}{\Gamma(\theta(p))} x \log ^{\theta(p)-1} x+O\left(x \log ^{\theta(p)-2} x\right)
$$

où

$$
g(1, \theta(p))=\prod_{p}\left\{\left(1-\frac{1}{p}\right)^{\theta(p)}\left(1+\frac{\theta(p)}{p}+\frac{\theta\left(p^{2}\right)}{p^{2}}+\cdots\right)\right\} .
$$

Dans l'équation de la preuve du Théorème 10 , posant $u=\theta(p) t^{f(p)}$, on obtient en utilisant le théorème de Selberg [6],

où

$$
\sum_{\substack{n \leq x \\ n=0\left(p_{0}\right)}} \theta(n)\left(\frac{u}{\theta(p)}\right)^{f(n) / f(p)}=\frac{g_{1}(1, u)}{\Gamma(u)} x \log ^{u-1} x+O\left(x \log ^{u-2} x\right)
$$

$$
g_{1}(1, u)=w(1+w)^{-1} \prod_{p}\left(1-\frac{1}{p}\right)^{u} \prod_{p}\left(1+\frac{u}{p}+\frac{\theta\left(p^{2}\right)\left(\frac{u}{\theta(p)}\right)^{f\left(p^{2}\right) / f(p)}}{p^{2}}+\cdots\right)
$$

et

$$
w=\left(\frac{\theta\left(p_{0}\right)\left(\frac{u}{\theta(p)}\right)^{f\left(p_{0}\right) / f(p)}}{p_{0}}+\frac{\theta\left(p_{0}^{2}\right)\left(\frac{u}{\theta(p)}\right)^{f\left(p_{0}^{2}\right) / f(p)}}{p_{0}^{2}}+\cdots\right) .
$$

D'où posant $u=\theta(p)$, on a

$$
\sum_{\substack{n \leq x \\ n=0\left(p_{0}\right)}} \theta(n)=\frac{g_{1}(1, \theta(p))}{\Gamma(\theta(p))} x \log ^{\theta(p)-1} x+O\left(x \log ^{\theta(p)-2} x\right)
$$

et alors on obtient le résultat demandé.

THÉORÈME 13

$$
\sum_{\substack{n \leq x \\ n=0\left(p_{n}\right)}} d(n) \sim \frac{2 p_{o}-1}{p_{0}^{2}} \sum_{n \leq x} d(n)
$$

et

$$
\sum_{\substack{n \leq x \\ n \neq 0\left(p_{0}\right)}} d(n) \sim\left(\frac{p_{0}-1}{p_{0}}\right)^{2} \sum_{n \leq x} d(n)
$$

où $p_{0}$ est un nombre premier fixe.

Preuve. Immédiat d'après le Théorème 12.

\section{Autres applications}

(i) Posons $f=\mu$ dans l'équation de la remarque (ii), alors pour $\sigma>1$

$$
\sum_{\substack{n=1 \\ n=0(k)}}^{\infty} \frac{\mu(n)}{n^{s}}=\frac{\mu(k)}{k^{s}} \prod_{p \mid k}\left(1-\frac{1}{p^{s}}\right)^{-1} \sum_{n=1}^{\infty} \frac{\mu(n)}{n^{s}}
$$


et

$$
\sum_{\substack{n=1 \\ n \neq 0(k)}}^{\infty} \frac{\mu(n)}{n^{s}}=\left\{1-\frac{\mu(k)}{k^{s}} \prod_{p \mid k}\left(1-\frac{1}{p^{s}}\right)^{-1}\right\} \sum_{n=1}^{\infty} \frac{\mu(n)}{n^{s}} .
$$

Prenons $\sigma>1$, dérivant les équations (4) et (5) par rapport à $s$, laissant $s \rightarrow 1^{+}$, et utilisant le fait (voir [3]) que

$$
\sum_{n=1}^{\infty} \frac{\mu(n) \log n}{n}=-1
$$

nous trouvons que

et

$$
\sum_{\substack{n=1 \\ n \equiv 0(k)}}^{\infty} \frac{\mu(n) \log n}{n}=-\frac{\mu(k)}{k} \prod_{p \mid k}\left(1-\frac{1}{p}\right)^{-1},
$$

$$
\sum_{\substack{n=1 \\ n \neq 0(k)}}^{\infty} \frac{\mu(n) \log n}{n}=\frac{\mu(k)}{k} \prod_{p \mid k}\left(1-\frac{1}{p}\right)^{-1}-1
$$

pour tout entier $k \in \mathbb{N}$.

(ii) Dans [3], on trouve les relations suivantes:

$$
\sum_{n=1}^{\infty} \frac{1-\Lambda(n)}{n}=2 \gamma
$$

et

$$
\sum_{n=1}^{\infty} \frac{1-\Lambda(n)}{n^{s}}=\zeta(s)\left(1+\sum_{n=1}^{\infty} \frac{\mu(n) \log n}{n^{s}}\right)
$$

où

$$
\Lambda(n)= \begin{cases}\log p & \text { si } \quad n=p^{\alpha}, \quad \alpha \in \mathbb{N} \\ 0 & \text { autrement }\end{cases}
$$

D'où

$$
\begin{aligned}
\sum_{n=1}^{\infty} \frac{1-\Lambda(n)}{n} & =\lim _{s \rightarrow 1^{+}} \sum_{n=1}^{\infty} \frac{1-\Lambda(n)}{n^{s}}=\lim _{s \rightarrow 1^{+}}\left(\frac{1+\sum_{n=1}^{\infty} \frac{\mu(n) \log n}{n^{s}}}{\sum_{n=1}^{\infty} \frac{\mu(n)}{n^{s}}}\right) \\
& =-\sum_{n=1}^{\infty} \frac{\mu(n) \log ^{2} n}{n}=2 \gamma .
\end{aligned}
$$

Encore une fois, prenons $\sigma>1$, dérivant l'équation (4) deux fois par rapport à $s$ et laissant $s \rightarrow 1^{+}$, on trouve

$$
\sum_{\substack{n=1 \\ n \equiv 0(k)}}^{\infty} \frac{1-\Lambda(n)}{n}=\frac{2 \mu(k)}{k} \prod_{p \mid k}\left(1-\frac{1}{p}\right)^{-1}\left(\gamma+\log k+\sum_{p \mid k} \frac{\log p}{p-1}\right)
$$

pour tout entier $k \in \mathbb{N}$. 
(iii) On sait que

$$
\sum_{n=1}^{\infty} \frac{\lambda(n)}{n^{s}}=\frac{\zeta(2 s)}{\zeta(s)}, \quad \sigma>1
$$

$\lambda$ étant la fonction de Liouville. En 1737, Euler communiqua, dans un article intitulé "Variae observationes circa series infinitas", le fait que

$$
\sum_{n=1}^{\infty} \frac{\lambda(n)}{n}=0
$$

Utilisant la remarque (i), on a,

$$
\sum_{\substack{n=1 \\ n=0(k)}}^{\infty} \frac{\lambda(n)}{n}=0 \quad \text { et } \sum_{\substack{n=1 \\ n \neq 0(k)}}^{\infty} \frac{\lambda(n)}{n}=0
$$

pour tout entier $k \in \mathbb{N}$.

Dérivant l'équation (6) pour $\sigma>1$ et laissant $s \rightarrow 1^{+}$, on obtient

$$
\sum_{n=1}^{\infty} \frac{\lambda(n) \log n}{n}=-\frac{\pi^{2}}{6} \text {. }
$$

Par la remarque (i), pour $\sigma>1$ on a

$$
\sum_{\substack{n=1 \\ n \equiv 0(k)}}^{\infty} \frac{\lambda(n)}{n^{s}}=\frac{\lambda(k)}{k^{s}} \sum_{n=1}^{\infty} \frac{\lambda(n)}{n^{s}} .
$$

Dérivant cette dernière équation pour $\sigma>1$ et laissant $s \rightarrow 1^{+}$,

$$
\sum_{\substack{n=1 \\ n=0(k)}}^{\infty} \frac{\lambda(n) \log n}{n}=-\frac{\pi^{2}}{6} \frac{\lambda(k)}{k}
$$

pout tout entier $k \in \mathbb{N}$, et de même

$$
\sum_{\substack{n=1 \\ n \neq 0(k)}}^{\infty} \frac{\lambda(n) \log n}{n}=-\frac{\pi^{2}}{6}\left(1-\frac{\lambda(k)}{k}\right)
$$

pour tout entier $k \in \mathbb{N}$.

\section{REFERENCES}

1. T. M. Apostol, Identities of the type $\sum f(n) \mu(n) n^{-s}$, Proceedings of the A.M.S., Vol. 40 (1973), 341-345.

2. T. M. Apostol, Note on series of the type $\sum f(n) \mu(n) n^{-s}$, Nordisk Matematisk Tidskrift, 23 (1975), 49-50.

3. R. Ayoub, An introduction to the analytic theory of numbers, Amer. Math. Soc., Mathematical Surveys, no. 10, 1963, (reprinted 1974).

4. R. Ayoub, Euler and the zeta formula, Amer. Math. Monthly, Vol. 81 (1974), 1067-1086. 
5. J. M. De Koninck, On a class of arithmetical functions, Duke Math. J. Vol. 39 (1972), 807-818.

6. Atle Selberg, Note on a paper by L. G. Sathe, J. Indian Math. Soc., Vol. 18 (1954), 83-87.

DÉPT, DE MATH.

UNIVERSITÉ DU QUÉBEC

Chicoutimi, Quebec 